\title{
Evaluation of Applied Axial Field Modulation Technique on ME Sensor Input Equivalent Magnetic Noise Rejection
}

\author{
Xin Zhuang, Marc Lam Chok Sing, Christophe Cordier, Sébastien Saez, Christophe Dolabdjian, Liangguo Shen, \\ Jie Fang Li, Menghui Li, and Dwight Viehland
}

\begin{abstract}
By using nonlinearity effects in magnetostrictive -piezoelectric laminated sensors, modulation techniques can transfer low-frequency signals to higher frequencies. Theory predicts that the transfer ability depends mainly on the amplitude of the carrier signal and the sensor nonlinearity. This was confirmed by our experiments. We present the first analysis on a ME noise model associated to a modulation technique. Furthermore, the overall equivalent magnetic noise was analyzed, and shown to be dominated only by the signal transfer ability and the output electrical noise level appearing around the carrier frequency.
\end{abstract}

Index Terms-Equivalent magnetic noise, laminated ME coefficient, magnetoelectric effect, nonlinearity effect.

\section{INTRODUCTION}

$\mathbf{M}$ AGNETOELECTRIC (ME) effects were found many decades ago in single phase materials [1]. This effect is enhanced several hundred times in magnetostrictive-piezoelectric laminated composite structures [2]. The amount of magnetic-to-electric energy that is transduced can be characterized by either the ME charge coefficient $(d Q / d H)$ or $\mathrm{ME}$ voltage coefficient, $(d V / d H)$ [3]. In general, this transduction capability of ME sensors is dominated by the piezoelectric and magnetostrictive coefficients in the laminated composites. Present ME laminated sensors can be classified into two general types, according to their operational modes: these are longitudinal or bending modes [4]. Longitudinal mode ME sensors vibrate center-symmetrically along the axial direction [5]. However, bending mode ME sensors vibrate with the help of a fixed point at one of its extremities [6]. Both types of sensors

X. Zhuang, M. L. C. Sing, C. Cordier, S. Saez, and, C. Dolabdjian are with the Groupe de Recherche en Informatique, Image, Automatique et Instrumentation de Caen (GREYC), CNRS UMR 6072-ENSICAEN and the University of Caen Basse Normandie, France 14050 Caen Cedex (e-mail: Xin.Zhuang @ greyc.ensicaen.fr; M.Lam@greyc.ensicaen.fr; christophe.cordier@unicaen.fr; sebastien.saez@unicaen.fr; christophe. dolabdjian@unicaen.fr).

L. Shen, J. F. Li, M. Li, and D. Viehland are with the Department of Materials Science and Engineering, Virginia Polytechnic Institute and State University, Blacksburg, VA 24061 USA (e-mail: shen99@ vt.edu; jiefang@mse.vt.edu; limh09@vt.edu; viehland@mse.vt.edu).

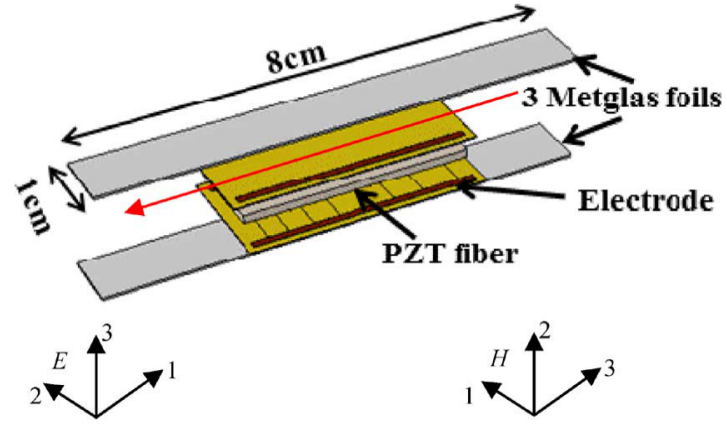

Fig. 1. Sketch view of the sensor with the associated three layers Metglas. The red arrow indicates the magnetic field sensed axis. The tensor axes of the system [cf. Eq. (1) and (2)] are also given for magnetic field $H$ [subscript: $m$ in (1) and (2)], and electric field $E$ (subscript: $p$ ). Both are applied in the direction 3 of their self-axis.

are fabricated with biphasic materials, namely magnetostrictive and piezoelectric material layers, which have high effective linear piezomagnetic coefficients $d_{m}$ and high piezoelectric coefficients $d_{p}$, respectively. In the real world, the physics of the system is not always linear. Because of nonlinearities in the piezomagnetic coefficients [7], the ME voltage or ME charge coefficients of these sensors will also be nonlinear. Such nonlinear effects will cause some signal distortion, especially when laminated ME sensors are used for detecting large amplitude signals. However, this nonlinear response can be used to transfer low-frequency signals to higher frequencies. Afterwards, the original signal can be recovered by proper demodulation techniques. In this manner, certain low-frequency perturbations can be eliminated with this spectrum-shift process.

\section{NONLINEARITY OF ME LAMINATED SENSOR}

Following [8], an equivalent model of the ME laminated sensor is proposed, based on constitutive piezoelectric, piezomagnetic and motion equations. The ME laminate composites used in this experiment (see illustration in Fig. 1) was a trilayer Metglas/PZT/Metglas fiber with a multi push-pull mode configuration. First, Kapton interdigitated or ID electrode layers were epoxied on both sides of a layer of five piezoelectric fibers bundled together that were each of dimensions $4 \mathrm{~cm} \times 0.2 \mathrm{~cm} \times 0.2 \mathrm{~cm}$.

The ID electrode pattern allows for symmetric poling of the fibers in a back-to-back pattern along their length axis. Second, three layers of Metglas foils (Vitrovac 7600F, Vitrovac Inc., Hanau, Germany) of dimensions $8 \mathrm{~cm} \times 1 \mathrm{~cm} \times 22 \mu \mathrm{m}$ were 


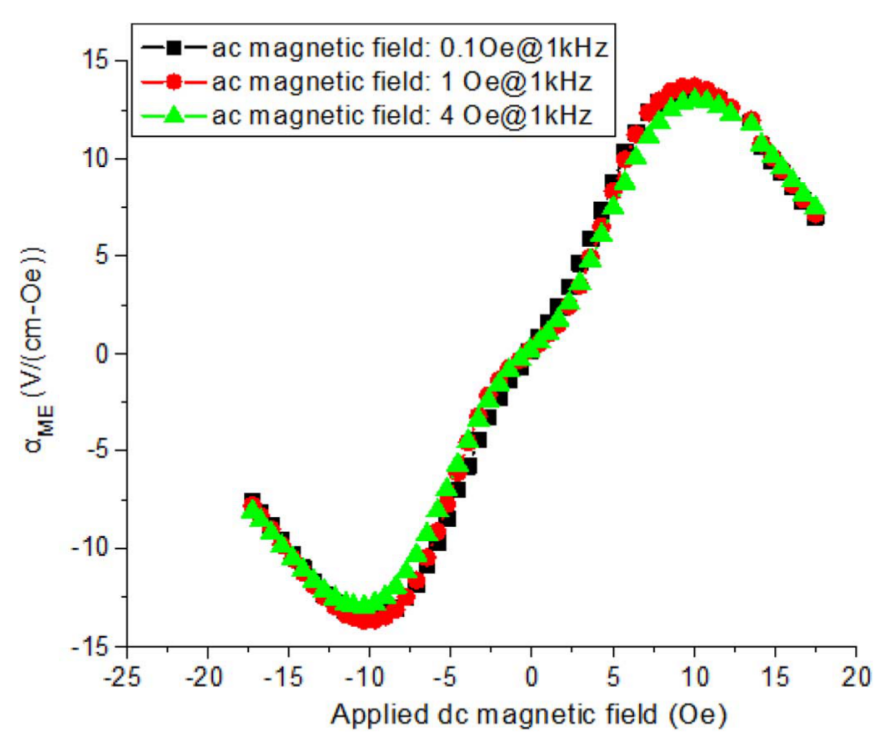

Fig. 2. ME voltage coefficient, $\alpha_{\mathrm{ME}}$, of a Metglas/PZT laminated sensor as a function of $\mathrm{dc}$ and ac magnetic bias. These measurements were made outside of a magnetically shielded chamber.

epoxied to each other. Finally, three-layer Metglas foils were epoxied on both sides of the piezoelectric fiber layer. Details about the laminate fabrication can be found in [5]. The dimensions of the completed laminates were approximately $0.5 \mathrm{~cm} \times$ $8.0 \mathrm{~cm} \times 1 \mathrm{~cm}$. Magnetic DC biases were then applied by permanent magnets placed at either end of the ME laminates. The ME voltage $(d V / d H)$ and ME charge $(d Q / d H)$ coefficients can be deduced from a 1D Mason's equivalent model [3], [8], [9] as

$$
\frac{d V}{d H} \approx \frac{n(1-n) d_{33, m} g_{31, p} t_{l a m}}{n s_{11, p}\left(1-k_{31, p}^{2}\right)+(1-n) s_{33, m}} \quad\left(\mathrm{~V} / \mathrm{A}-\mathrm{m}^{-1}\right)
$$

or

$\frac{d Q}{d H} \approx \frac{n d_{33, m} h_{31, p} \varepsilon_{33}^{S} s_{11, p} l w}{n s_{11, p}+(1-n) s_{33, m}\left(1-s_{11, p} h_{31, p}^{2} \varepsilon_{33}^{S}\right)}\left(\mathrm{C} / \mathrm{A}-\mathrm{m}^{-1}\right)$

where $s_{11, p}$ and $s_{33, m}$ are the elastic compliances of the piezoelectric and magnetostrictive layers, $d_{33, m}$ the effective linear longitudinal piezomagnetic coefficient, $h_{31, p}$ the piezoelectric constant, $g_{31, p}$ the transverse piezoelectric voltage coefficient, $k_{31, p}$ and $\varepsilon_{33}^{S}$ the piezoelectric coupling coefficient and dielectric constant at constant stress, $k_{31, p}$ the piezoelectric constant, $n$ the thickness ratio of the piezoelectric and magnetostrictive layers, $l$ and $w$ the length and width of the laminate, and $t_{\text {lam }}$ the total thickness of the laminate. These formulas are nonlinear since the piezomagnetic or piezoelectric coefficients involved are not linear.

Figs. 2 and 3 show the ME voltage coefficient $\left(\alpha_{\mathrm{ME}}\right)$ as a function of applied dc bias $H_{\mathrm{dc}}$ for an applied ac magnetic field $H_{\mathrm{ac}}$ of different amplitude at $1 \mathrm{kHz}$ for Metglas $/ \mathrm{Pb}(\mathrm{Zr}, \mathrm{Ti}) \mathrm{O}_{3}$ - fiber/Metglas (or Metglas/PZT/Metglas) and Metglas/Pb( $\left.\mathrm{Mg}_{1 / 3} \mathrm{Nb}_{2 / 3}\right) \mathrm{O}_{3}-$ $30 \mathrm{at} \% \mathrm{PbTiO}_{3} /$ Metglas single-crystal fiber (or Metglas/PMN-PT/Metglas) three-layer laminates. These laminates were fabricated following [5]. The PZT fibers were obtained

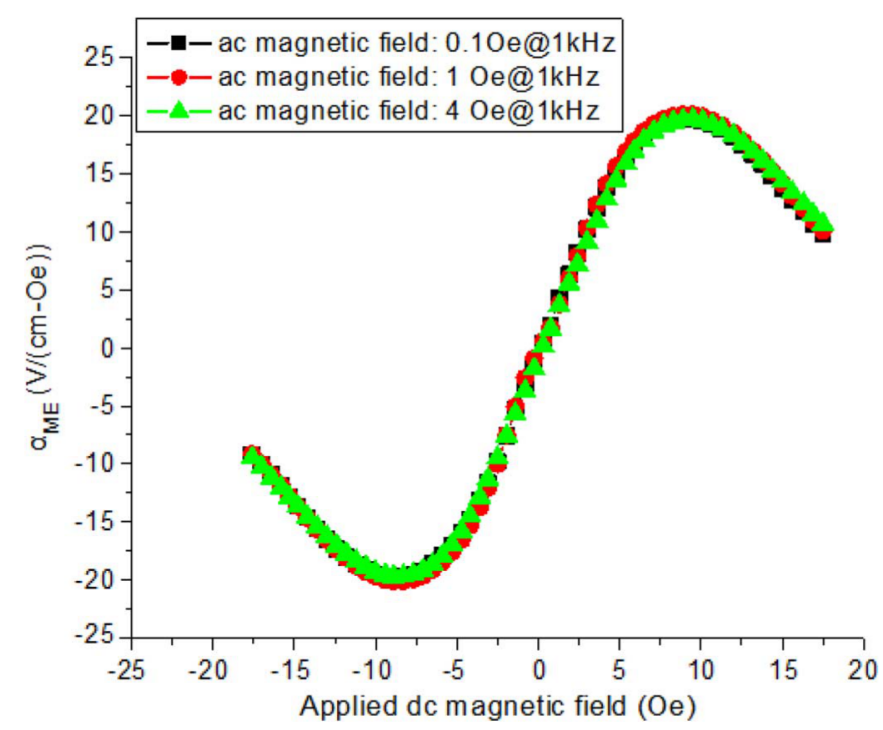

Fig. 3. ME voltage coefficient for a single crystal ME sensor as a function of the dc and ac magnetic bias. These measurements were made outside of a magnetically shielded chamber.

from CTS (Albuquerque, NM) and the PMN-PT ones from the Shanghai Institute of Ceramics Chinese Academy of Science (Shanghai, China).

Piezoelectric materials are known to have significant nonlinearities in their piezoelectric coefficients, which are believed to be due to contributions from domain wall dynamics [10]. However, presently, this phenomenon is not well understood in term of basic physics and how it contributes to the materials properties. Here, in this investigation, we have observed under dc magnetic bias a nonlinear magnetoelectric effect. The observed nonlinearity is largest under a particular $\mathrm{H}_{\mathrm{dc}}$ bias (around \pm 4 and \pm 2 Oe for the metglas/PZT and metglas/single crystal sensors, respectively): which would be the best operational point for our cross or axial modulation studies [11].

\section{TheORETICAL ANALYSIS OF NONLINEARITY MODUlation}

\section{A. Spectrum Transfer Ability}

As for any nonlinear system [12], the induced charge response of ME laminated sensors can be written as a Taylor expansion series

$$
Q(t)=a_{0}+a_{1} B(t)+a_{2} B^{2}(t)+a_{3} B^{3}(t)+\ldots
$$

where $Q(t)$ is the induced electric charge, $a_{i}(i=0$ to $n)$ are the $i^{\text {th }}$ coefficients, and $B(t)$ the applied magnetic signal. If we consider that the applied magnetic signal $B(t)$ is composed of a low-frequency magnetic signal $B_{0}(t)$ and an excitation carrier $B_{t}(t)$, (3) can be rewritten as

$$
Q(t) \approx a_{1}\left(B_{0}(t)+B_{t}(t)\right)+a_{2} B_{0}(t) B_{t}(t)+\ldots
$$

Thus, we can get a cross modulation term $a_{2} B_{t}(t) B_{0}(t)$ around the carrier frequency, $f_{e} . a_{1}$ is the equivalent charge coefficient for the applied signals $B_{0}(t)$ and $B_{t}(t)$ and $a_{2}$ represents a new cross modulation coefficient for $B_{0}(t) B_{t}(t)$, which we name the low-frequency signal transfer ability. As for any modulation system, the carrier frequency $f_{e}$ is much higher than the 


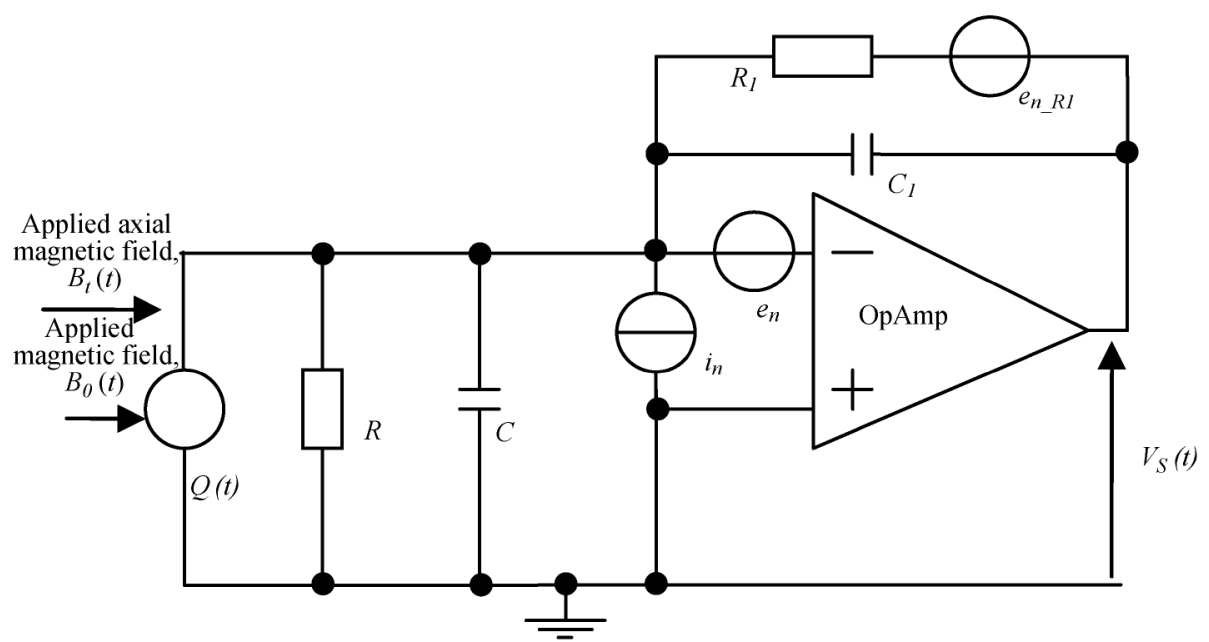

Fig. 4. Schematic view of the modulated current charge preamplifier electronics with classical associated charge amplifier noise sources. We define the sensor impedance as $Z=R / / C$ and the generated charge source $Q(t)$.

frequency $f_{0}$ of the applied signal. With the hypothesis that $f_{e} \gg f_{0}$, we can define in the harmonic regime that

$$
\left\{\begin{array}{l}
a_{1}=\alpha_{\mathrm{ME}} \\
a_{2} B_{t}(t)=\alpha_{\mathrm{ME}, f_{e}} B_{t} \cos \left(2 \pi f_{e} t\right)
\end{array}\right.
$$

where $B_{t}$ is the peak amplitude of the sinusoidal excitation carrier, $B_{t}(t)=B_{t} \cos \left(2 \pi f_{e} t\right)$. Notice that the units of $\alpha_{\mathrm{ME}}$ and $\alpha_{\mathrm{ME}, f_{e}}$ are given in $(\mathrm{C} / \mathrm{T})$ and $\left(\mathrm{C} / \mathrm{T}^{2}\right)$, respectively.

In the temporal regime, with the help of a classical current charge amplifier circuit [6] as shown in Fig. 3, the output voltage versus an applied magnetic field can be given as

$$
\begin{array}{r}
V_{s}(t) \approx\left(\left(\alpha_{\mathrm{ME}} B_{t}+\alpha_{\mathrm{ME}, f_{e}} B_{t} B_{0}(t)\right) \cos \left(2 \pi f_{e} t\right)\right) \\
\otimes F^{-1}\left(\frac{R_{1}}{1+j 2 \pi f R_{1} C_{1}}\right)
\end{array}
$$

where $F^{-1}$ is the inverse Fourier function and $\otimes$ is the convolution product.

By taking the Fourier Transform, the output voltage can be written as

$$
\begin{aligned}
V_{s}(f) \approx & \left(\alpha_{\mathrm{ME}} B_{t} \mathrm{D}-\operatorname{Delta}(f)+\alpha_{\mathrm{ME}, f_{e}} B_{t} B_{0}(f)\right) \\
& \times\left(\frac{\mathrm{D}-\operatorname{Delta}(f-f)_{e}+\mathrm{D}-\operatorname{Delta}\left(f+f_{e}\right)}{2}\right) \\
& \times\left(\frac{R_{1}}{1+j 2 \pi f R_{1} C_{1}}\right)
\end{aligned}
$$

where D - Delta $(f)$ represents the Dirac Delta function. This can be simplified to

$$
V_{s}(f) \approx \frac{\alpha_{\mathrm{ME}, f_{e}} B_{t}}{C_{1}}\left(\frac{B_{0}(f-f)_{e}+B_{0}\left(f+f_{e}\right)}{2}\right)
$$

for $f_{e} \gg\left(1 / 2 \pi R_{1} C_{1}\right)$ and also be written as

$$
V_{s}(t) \approx \frac{\alpha_{\mathrm{ME}, f_{e}} B_{t}}{C_{1}} \cos \left(2 \pi f_{e} t\right) B_{0}(t) .
$$

By using a classical demodulation technique with a sine wave product detector of $2 \cos \left(2 \pi f_{e} t\right)$ and by filtering off the high- frequency components, the original signal can thus be recovered. Therefore, the output transfer function in (Volt/Tesla) after demodulation and filtering is

$$
\left|T_{r_{-} A F}(f)\right| \approx \frac{\alpha_{\mathrm{ME}, f_{e}} B_{t}}{C_{1}} .
$$

\section{B. Noise Analysis}

We treat noise as a perturbation of a classical charge current amplifier [6]. In our model, if we consider Johnson, amplifier [13] and sensor dielectric loss [4] noise sources as intrinsic noise and the environmental noise as extrinsic, then the block diagram of Fig. 4 can be used to represent the noise model for ME laminated magnetic sensors.

For our ME sensors, an external magnetic signal $B_{t}(t)$ excites the sensor near its magnetoelastic resonant frequency $f_{\text {res }}$ of $\sim 27 \mathrm{kHz}$. The reference signal $B_{0}$ is the low-frequency information signal. $\alpha\left(t, B_{\mathrm{dc}}, B\right)$ is the charge transfer function coefficient with units of C/T. $b_{n_{-} R}, b_{n_{-} P o l}, b_{n}(\mathrm{~T} / \sqrt{ } \mathrm{Hz})$ are the Johnson noise, thermal polarization noise and intrinsic magnetic sensor noise sources, respectively. All contribute to the total equivalent magnetic noise of the ME laminated sensor. $q_{n_{-} v i b}$ is an external vibration noise, which is effectively seen as a thermal charge noise.

The output voltage transfer function of the charge amplifier shown in Fig. 4 versus the applied magnetic field in (Volt/Tesla) can be written [6] as

$$
\begin{aligned}
T_{r}(f) & =\frac{\alpha_{\mathrm{ME}}(f)}{C_{1}}\left(\frac{j(2 \pi f) R_{1} C_{1}}{1+j(2 \pi f) R_{1} C_{1}}\right) \\
& \left.\approx \frac{\alpha_{\mathrm{ME}}(f)}{C_{1}}\right|_{f \gg\left(2 \pi R_{1} C_{1}\right)^{-1}} .
\end{aligned}
$$

The total output voltage noise spectral density in $(\mathrm{V} / \sqrt{ } \mathrm{Hz})$ can then be written as

$$
\begin{aligned}
e_{n_{-} T}^{2}(f)= & \left|Z_{1}\right|^{2}\left[i_{n_{-} R_{1}}^{2}(f)+i_{n}^{2}(f)+i_{n_{-} v i b}^{2}(f)\right] \\
& +\left|1+\frac{Z_{1}}{Z}\right|^{2} e_{n}^{2}(f)+\left|T_{r}(f)\right|^{2} b_{n_{-} \text {sensor }}^{2}(f)
\end{aligned}
$$




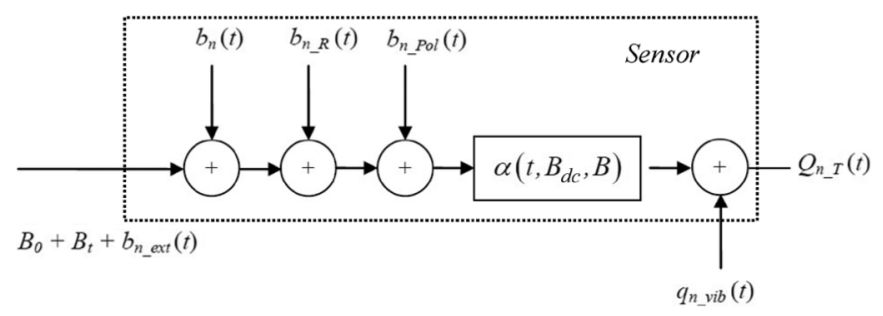

Fig. 5. Proposed noise model of the ME laminated sensor with associated noise sources.

where $i_{n_{-} R 1}^{2}(f)=e_{n_{-} R 1}^{2}(f) / R_{1}^{2}, \quad i_{n_{-} v i b}^{2}(f)=$ $(2 \pi f)^{2} q_{n \_v i b}^{2}(f), Z_{1}=R_{1} /\left(1+j(2 \pi f) C_{1} R_{1}\right), Z=$ $R /(1+j(2 \pi f) C R) . b_{n_{-} s e n s o r}(f)$ and $q_{n_{-} v i b}(f)$ are the equivalent magnetic field noise of the sensor and equivalent charge noise induced by mechanical vibration (i.e., elastic and/or acoustic waves), respectively. Thus, the expected total equivalent input magnetic noise power spectral density of the sensor in $\left(\mathrm{T}^{2} / \mathrm{Hz}\right)$ is

$$
\begin{aligned}
b_{n_{-} T e q}^{2}(f)= & \frac{e_{n_{-} T}^{2}(f)}{\left|T_{r}\right|^{2}} \\
= & b_{n_{-} R_{1}}^{2}(f)+b_{n_{-} i n}^{2}(f)+b_{n_{-} e n}^{2}(f) \\
& +b_{n_{-} v i b}^{2}(f)+b_{n_{-} s e n s o r}^{2}(f) .
\end{aligned}
$$

From our model (cf. Fig. 5), we can write

$$
b_{n_{-} \text {sensor }}^{2}(f)=b_{n_{-} R}^{2}(f)+b_{n}^{2}(f)+b_{n_{-} P o l}^{2}(f) .
$$

If $R$ is not considered as having an infinite value, we can obtain

$$
\begin{aligned}
b_{n_{-} T e q}^{2}(f) \approx & \frac{1}{\alpha_{\mathrm{ME}}^{2}(f)}\left[\frac{1}{(2 \pi f)^{2}}\left(i_{n}^{2}(f)+\frac{4 k_{b} T}{R_{1}}\right)\right. \\
& \left.+q_{n_{-} v i b}^{2}(f)\right] \\
& +\frac{1}{\left|T_{r}(f)\right|^{2}}\left|1+\frac{Z_{1}}{Z}\right|^{2} e_{n}^{2}(f)+b_{n_{-} R}^{2}(f) \\
& +b_{n}^{2}(f)+b_{n_{-} P o l}^{2}(f)
\end{aligned}
$$

where

$$
\begin{aligned}
b_{n_{-} P o l}^{2}= & 2 \pi f 4 k_{b} T C \tan (\delta(f))\left(\frac{R_{1}^{2}}{1+\left(2 \pi f R_{1} C_{1}\right)^{2}}\right) \\
& \times \frac{1}{\left|T_{r}(f)\right|^{2}} \\
= & \frac{4 k_{b} T C}{2 \pi f \alpha_{\mathrm{ME}}^{2}(f)} \tan ((\delta(f)))
\end{aligned}
$$

and where $C=\varepsilon_{33}\left(l_{w} / t_{p}\right)$ and $\tan (\delta(f))$ are the capacitance and dielectric loss factor as function of frequency. $l, w$ and $t_{p}$ are the length, width and thickness of the piezoelectric layer respectively. The external magnetic noise spectral power density $b_{n}^{2}$ represents any unknown external noise superimposed to the magnetic signals.

The Johnson noise power spectral density of the ME laminated sensor is

$$
\begin{aligned}
b_{n_{-} R}^{2}(f) & =\frac{4 k_{b} T}{R}\left(\frac{R_{1}^{2}}{1+\left(2 \pi f R_{1} C_{1}\right)^{2}}\right) \frac{1}{\left|T_{r}(f)\right|^{2}} \\
& =\frac{1}{(2 \pi f)^{2} \alpha_{\mathrm{ME}}^{2}(f)} \frac{4 k_{b} T}{R} .
\end{aligned}
$$

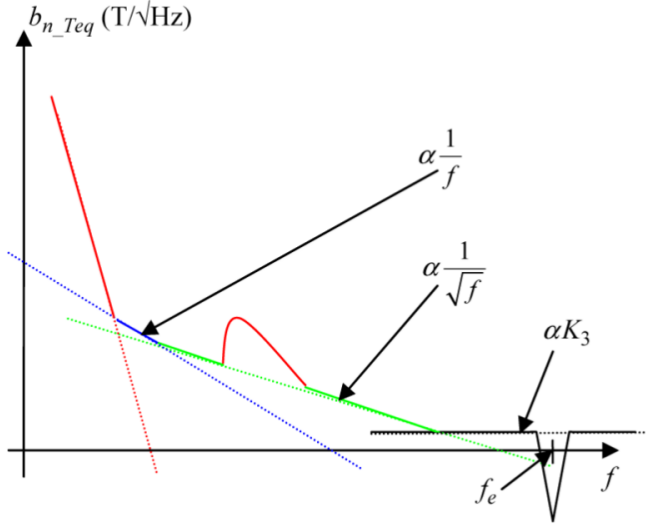

Fig. 6. An example of the expected equivalent magnetic noise behavior given by the model and experimental response. Color source contributions are detailed in equation (17). The red part of the curves are induced by vibrational noise sources. The latter have been confirmed by cross correlation technique measurements.

Thus, we obtain the full equivalent input magnetic noise equation as

$$
\begin{array}{r}
b_{n_{-} T e q}^{2}(f)=\frac{1}{\alpha_{\mathrm{ME}}^{2}(f)}\left[\frac { 1 } { 4 \pi ^ { 2 } f ^ { 2 } } \left(i_{n}^{2}(f)+\frac{4 k_{B} T}{R_{1}}+\frac{4 k_{B} T}{R}\right.\right. \\
\left.\quad+\frac{e_{n}^{2}(f)}{R_{1}}+\frac{e_{n}^{2}(f)}{R}\right) \\
+\frac{4 k_{b} T C}{2 \pi f} \tan (\delta(f)) \\
+\left(C_{1}+C_{i}\right)^{2} e_{n}^{2}(f)+q_{n_{-} v i b}^{2}(f) \\
\left.+b_{n}^{2}(f)\right] \\
\approx \frac{1}{\alpha_{\mathrm{ME}}^{2}(f)}\left[\frac{K_{1}}{f^{2}}+\frac{K_{2}}{f}+q_{n_{-} v i b}^{2}(f)+K_{3}\right]
\end{array}
$$

where $K_{1}, K_{2}$, and $K_{3}$ are constant terms and the color source contributions to the equivalent magnetic noise are represented in Fig. 6.

From these calculated and experimental observations, we can find, with regards to our hypothetical model, that the equivalent magnetic noise density has different regimes each leading to a different slope. For the different current noise sources $\left(i_{n}, R_{1}, R \ldots\right)$, we have an $1 / f$ behavior. The dielectric loss noise gives a $1 / f^{1 / 2}$ slope. The flat floor at higher frequencies is mainly induced by the equivalent input noise voltage, $e_{n}$, of the amplifier. We assume that the low-frequency noise is mainly that induced by vibrational sources. This effect has been confirmed by further cross correlation measurements. It varies as $1 / f^{2}$ for our experimental and environmental setup.

Thus, the expected equivalent noise sources for our model can be summarized, as given in Fig. 6. Due to the increase in transfer coefficient [12], we can notice that the equivalent magnetic noise is lower at the resonant frequency and yields to the highest expected signal-to-noise ratio by cross modulation technique.

\section{EXPERIMENTAL MEASUREMENTS AND DISCUSSIONS}

\section{A. Measurement Setup}

A reference signal $B_{0}$ was generated by using a voltage generator in series with a resistor and a pair of Helmholtz coils with 


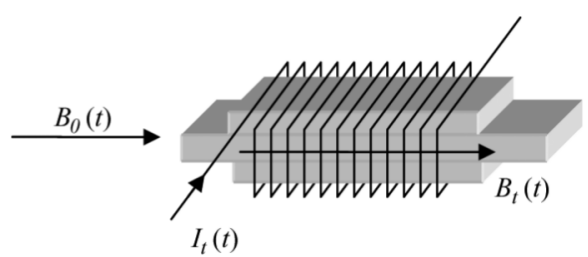

Fig. 7. Schematic view of the sensor with its associated coil. We designate the excitation signal as $B_{t}(t)$ and the reference signal as $B_{0}(t)$. The current $I_{t}(t)$ is used to generate the applied axial field, $B_{t}(t)$.

a transfer coefficient of $5.37 \times 10^{-4} \mathrm{~T} / \mathrm{A}$. An axial magnetic field $B_{t}(t)$ of high frequency was used as an excitation signal. This excitation signal was generated by a second coil, closely wounded around the sensor, as shown in Fig. 7, with a transfer coefficient of about $11 \times 10^{-3} \mathrm{~T} / \mathrm{A}$.

In order to maximize the signal transfer ability, the frequency of excitation signal was tuned at the sensor's resonant frequency of $\sim 27 \mathrm{kHz}$. We used a low noise LTC6240 amplifier from Linear Technology having an equivalent input voltage noise $e_{n}=7 \mathrm{nV} / \sqrt{ } \mathrm{Hz}$ with a low corner frequency $f_{c}=10 \mathrm{~Hz}$ and an equivalent input current noise of $i_{n}=0.6 \mathrm{fA} / \sqrt{ } \mathrm{Hz}$. A feedback resistor $R_{1}$ and capacitor $C_{1}$ of $10 \mathrm{G} \Omega$ and $100 \mathrm{pF}$, respectively, were used as shown in Fig. 4. The internal resistance $R$ and capacitance $C$ of the sensor were around $20 \mathrm{G} \Omega$ and $1 \mathrm{nF}$, respectively.

For our experiments, an applied carrier signal of large amplitude was necessary, thereby, requiring a high dynamic range of detection for the nonlinear effects to be observed. In order to correctly measure the reference signal's spectrum, a process of carrier frequency suppression was necessary and therefore, we used a differential preamplifier. The detected signal was applied to the amplifier noninverting input and the inverting input was connected to a voltage generator which was phase-locked to the carrier frequency. The preamplifier bandwidth ranged from $100 \mathrm{~Hz}$ to $300 \mathrm{kHz}$ with a gain of 500 . Thus, by adjusting the amplitude and phase of the generator at the inverting input, we can minimize the amplitude of the carrier at the preamplifier output. The reference information signal can then be optimally demodulated by a lock-in amplifier (SRS SR-850).

\section{B. Transfer Function Measurements}

In our experiments, a reference sinusoidal signal of $3.8 \mathrm{nT} \mathrm{T}_{\mathrm{rms}}$ was applied at $2 \mathrm{~Hz}$, representing the information signal. The test sensor was excited by a magnetic field $B_{t}$ of about a microTesla in amplitude. As expected, the reference signal spectrum appeared around the carrier frequency, as shown in Fig. 8(a). With the help of a spectrum analyzer, the RMS value $V_{1}$ of the sensor response to the reference signal in the modulated signal spectrum was determined. It was found to be about $5 \mu \mathrm{V}_{\text {rms }}$ at both $26998 \mathrm{~Hz}$ and $27002 \mathrm{~Hz}$. This voltage value corresponds to the calculated term of $V_{1}=\left(B_{0} B_{t} \alpha_{M E, f_{e}} / 2 C_{1}\right)$. Thus, by using a classical demodulating technique (cf. Section III-A), the expected magnetic field transfer function can be written as

$$
\left|T_{r_{-} A F}(f)\right| \approx \frac{\alpha_{M E, f_{e}} B_{t}}{C_{1}}=\frac{2 V_{1}}{B_{0}}
$$
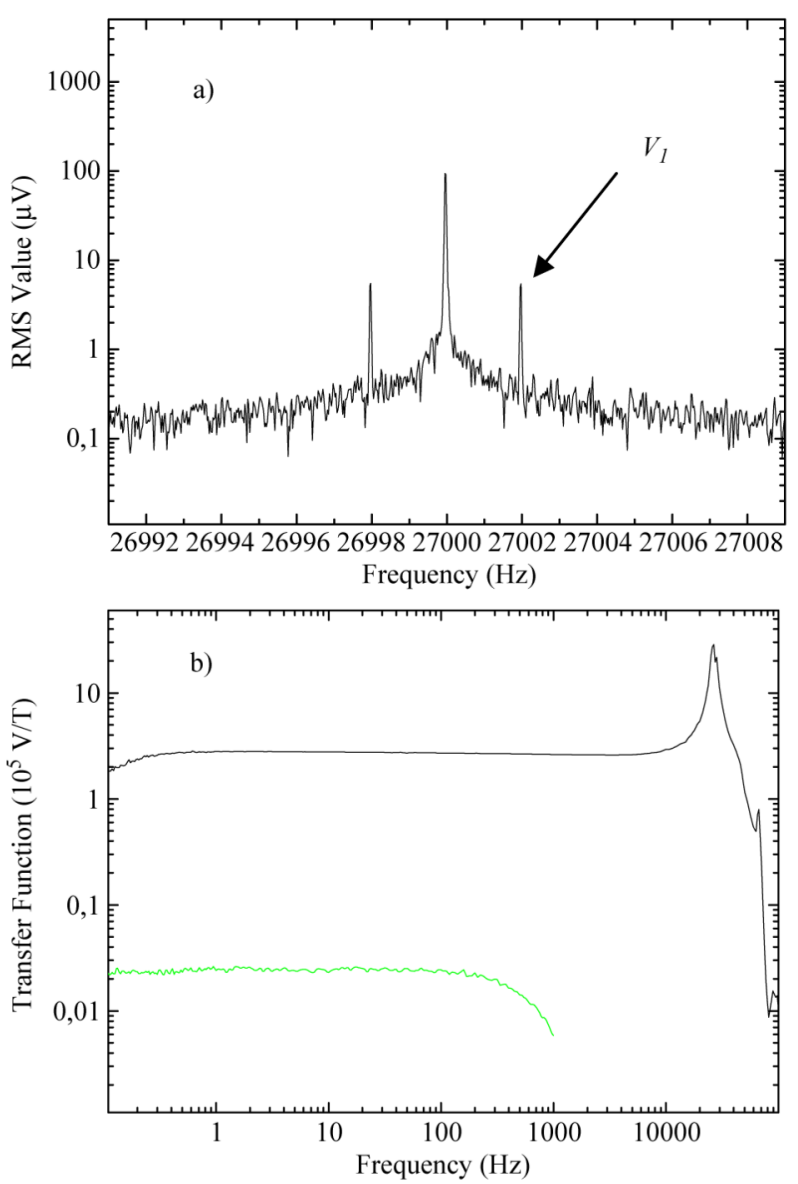

Fig. 8. (a) Modulated signal spectrum amplitude at the charge amplifier output $V_{s}(t)$. (b) Magnetic field transfer functions with nonlinearity modulation technique (green curve) and without modulation (black curve).

following (9). The theoretical magnetic field transfer function, calculated from this expression is about $2630 \mathrm{~V} / \mathrm{T}$. Furthermore, Fig. 8(b) gives the measured transfer function for the magnetic reference signals, which was about $2500 \mathrm{~V} / \mathrm{T}$ with a bandwidth from DC to $1 \mathrm{kHz}$. The gain value of the transfer function has to be normalized by the gain of preamplifier and lock-in amplifier (SRS SR 850). Our results agree quite well with the predicted values.

\section{Output Electrical Noise and Equivalent Magnetic Noise Measurements}

The output electrical noise was expected to be "white" over the measured bandwidth. However, when driven by the excitation signals, we found an asymmetrical increase of the noise level near the carrier frequency. This finding can clearly be seen in Fig. 9(a). This results in the demodulated noise spectra level not being flat, as can be seen in Fig. 9(b). This increase of the noise level near the carrier frequency could result from carrier versus demodulator phase jitter or dielectric loss noise of the ME laminated sensor. Only, the latter was considered in our theoretical analysis and it explains quite well the sensor behavior. Furthermore, by analyzing the slope of the measured curve [cf. green curve in Fig. 9(b)], we observed that the vibrational noise $q_{n_{-} v i b}(t)$ was rejected by the modulation process. 

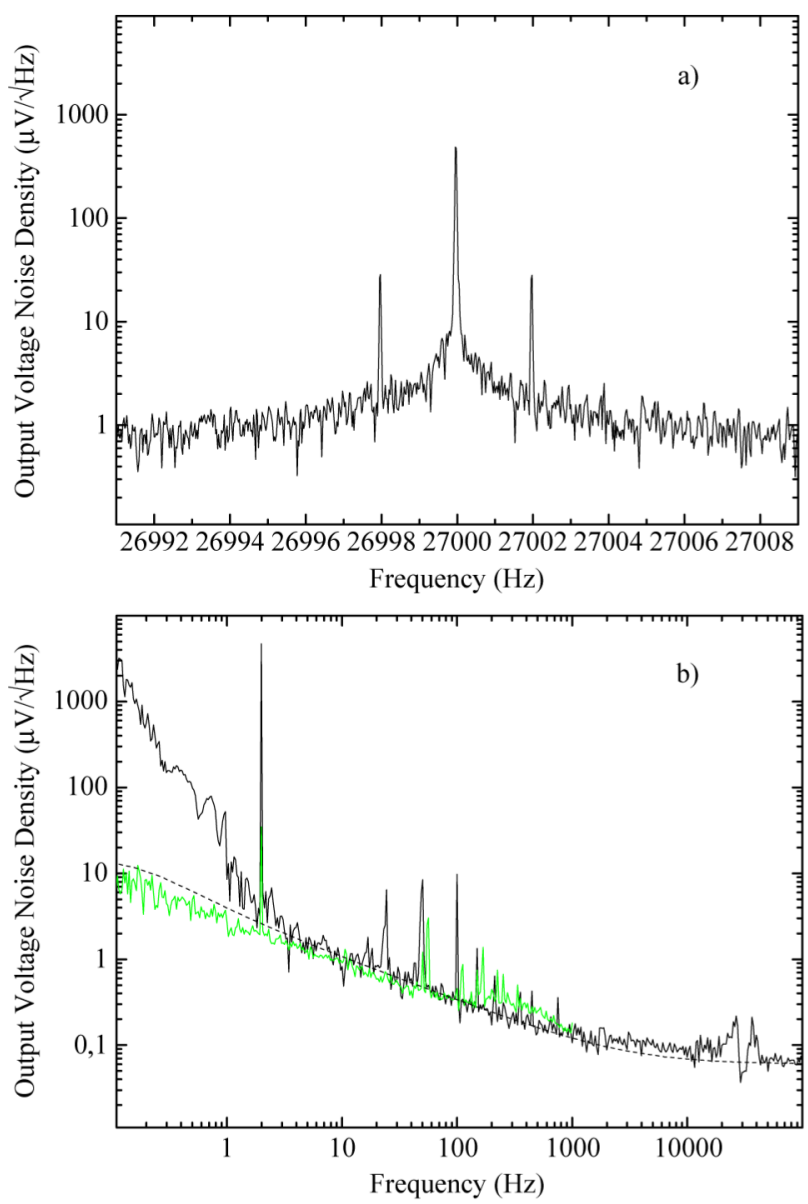

Fig. 9. Output electrical noise level (a) around the carrier frequency and (b) after demodulation by a lock-in amplifier. In curve (b), the black and green curves are the measured noise level (without cross modulation) and the one obtained by the cross modulation techniques, respectively. The dashed black line is the expected value versus the given $e_{n_{-} T}(f)$ noise model [cf. (11)].

If we divide the output electric noise curve by the corresponding transfer function, we obtain the equivalent input magnetic noise. The equivalent input magnetic noise level for our cross modulation technique is represented in Fig. 10. The solid and dashed lines are, respectively, the measured and the simulated results. Indeed, by using a classical demodulating technique, the latter can be written as [14]

$$
\begin{aligned}
\left|b_{n_{-} A F}(f)\right| \approx & \frac{\sqrt{2} e_{n}\left(f_{e}\right)+e_{n_{-} \text {sensor }}(f)}{\left|T_{r_{-} A F}\left(f_{e}\right)\right|} \\
\approx & \frac{1}{B_{t} \alpha_{\mathrm{ME}, f_{e}}} \\
& \times \sqrt{\frac{\left(4 k_{b} T C_{i}\right) \operatorname{Tan}(\delta(f))}{2 \pi f}+2 C_{1} e_{n}^{2}\left(f_{e}\right)}
\end{aligned}
$$

In Fig. 11, we compare the equivalent input magnetic noise without modulation to that observed with our nonlinear modulation technique.

For the unmodulated measurement (black curve in Fig. 11), an environmental vibrational noise can be observed at low frequencies $(<2 \mathrm{~Hz})$. At very low-frequency $(<0.2 \mathrm{~Hz})$, the equivalent magnetic noise obtained by the nonlinear modulation technic is lower than the direct measurement.

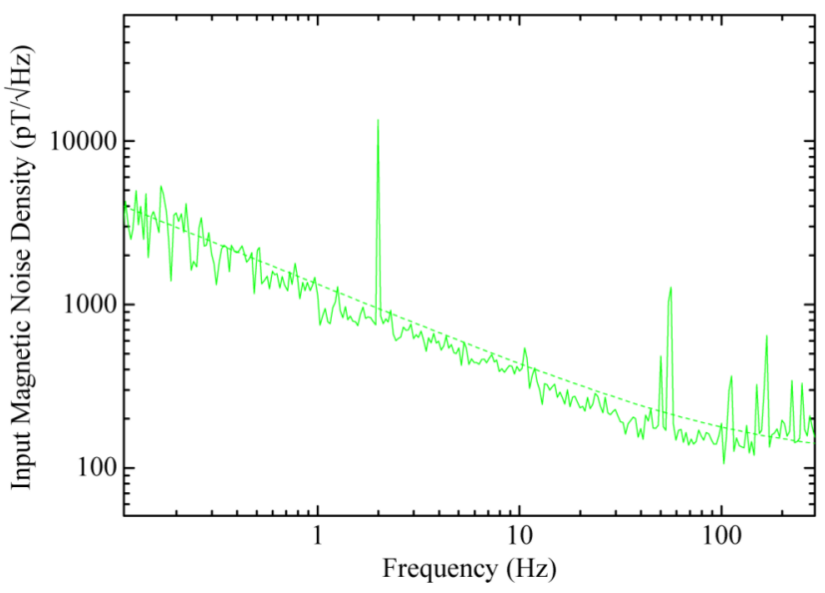

Fig. 10. Equivalent input magnetic noise with cross modulation techniques. Solid line is the measurement curve, and dashed line is the simulation curve given by (17) with parameters as follows: $C=1 \mathrm{nF}, \operatorname{Tan}(\delta)=0.055$, $R=\infty$.

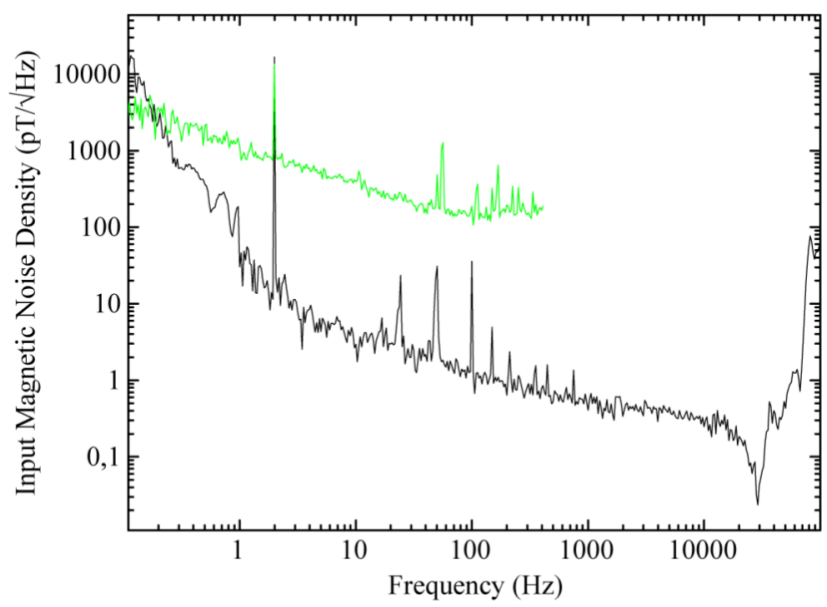

Fig. 11. Comparison of equivalent input magnetic noise, with and without modulation, green and black curves, respectively.

\section{CONCLUSION}

Nonlinearity of the ME coefficients of laminated ME sensors is a direct consequence of the piezoelectric and piezomagnetic nonlinearities of the respective individual layers. This ME nonlinear effect allows for a nonlinear modulation technique which transfer only low-frequency equivalent magnetic signals to higher frequencies. So, low-frequency signals will not suffer from low-frequency interferences - such as noise vibration!

We have analyzed the signal transfer ability, as well as noise levels, with this nonlinear modulation technique for three layer Metglas/PZT/Metglas and Metglas/PMN-PT/Metglas laminates. Experimental measurements confirm the predicted behavior. In the future, enhancement of the nonlinear transfer ability is an important goal for this modulation method, as it would foster the ability to reject low-frequency environmental vibrational noises, allows DC signal measurement and, thus, to lower the equivalent magnetic noise floor. Further analyses have to be done to improve the knowledge of the intrinsic sensor noise source, their possible rejection and the limit of the proposed technique. 


\section{REFERENCES}

[1] L. D. Landau and E. M. Lifshitz, Electrodynamics of Continuous Media. Oxford, U.K.: Pergamon, 1960, p. 119.

[2] J. Ryu, A. V. Carazo, K. Uchino, and H. E. Kim, "Magnetoelectric properties in piezoelectric and magnetostrictive laminate composites," Jpn. J. Appl. Phys., vol. 40, pp. 4948-4951, 2001.

[3] C.-W. Nan, "Magnetoelectric effect in composites of piezoelectric and piezomagnetic phases," Phys. Rev. B., vol. 50, no. 9, pp. 6082-6088, 1994.

[4] Z. Xing, J. Zhai, J. F. Li, and D. Viehland, "Investigation of external noise and its rejection in magnetoelectric sensor design," J. Appl. Phys., vol. 106, p. 024512, 2009.

[5] J. Das, J. Gao, Z. Xing, J. F. Li, and D. Viehland, "Enhancement in the field sensitivity of magnetoelectric laminate heterostructures," Appl. Phys. Lett., vol. 95, p. 092501, 2009.

[6] Z. Xing, J. Zhai, S. Dong, J. F. Li, D. Viehland, and W. Odendaal, "Modeling and detection of quasi-static nanotesla magnetic field variations using magnetoelectric laminate sensors," Meas. Sci. Technol., vol. 19, p. 015206, 2008.

[7] S. Dong, A. J. Y. Zhai, J. Li, and D. Viehland, "Magnetoelectric effect in Terfenol- $\mathrm{D} / \mathrm{Pb}(\mathrm{Zr}, \mathrm{TiO})(3) /$ mu-metal laminate composites," Appl. Phys. Lett., vol. 89, no. 12, p. 122903, 2006.

[8] S. Dong, J. F. Li, and D. Viehland, "Longitudinal and transverse magneto-electric voltage coefficients of piezomagnetic/piezoelectric laminate composites, I. Theory," IEEE Trans. Ultrasonics, Ferroelectrics and Frequency Control, vol. 50, no. 10, pp. 1253-1261, 2003.

[9] X. Zhuang, GREYC internal report, July 2010.

[10] M. Kläui, J. Phys. Condensed Matter, vol. 20, p. 313001, 2008.

[11] L. Shen, M. Li, J. Gao, Y. Shen, J. F. Li, D. Viehland, X. Zhuang, M. L. C. Sing, C. Cordier, S. Saez, and C. Dolabdjian, "Magnetoelectric nonlinearity in Metglas/Pb(Zr,Ti)O3 and Metglas/ $\mathrm{Pb}(\mathrm{Mg} 1 / 3, \mathrm{Nb} 2 /$ 3) $\mathrm{O} 3 \mathrm{PbTiO} 3$ laminate composites," $J$. Appl. Phys., submitted for publication.

[12] T. W. S. Chow, H. Tan, and Y. Fang, Wiley Encyclopedia of Electrical and Electronics Engineering. New York: Wiley, Dec. 20, 2001.

[13] X. Zhuang, M. L. C. Sing, C. Cordier, S. Saez, C. Dolabdjian, J. Das, J. Gao, J. F. Li, and D. Viehland, "Analysis of noise in Magneto-Electric thin layer composites used as magnetic sensors," IEEE Sensors J., submitted for publication.

[14] M. L. C. Sing, C. Dolabdjian, C. Gunther, D. Bloyet, and J. Certenais, "Formal presentation and comparative study of low-frequency noise reduction techniques for direct current superconducting quantum interference devices," Rev. Sci. Instr., vol. 67, no. 3, pp. 796-804, 1996. 